\title{
Improvement of Etching Method of Macroscopic Metallographic Examination Based on TRIZ Theory
}

\author{
Jianjun Yu, Xucheng Rong \\ School of Business Administration, South China University of Technology, Guangzhou, 510640, \\ China \\ yujj@scut.edu.cn
}

Keywords: Macro examination, metallographic, improvement, TRIZ

\begin{abstract}
Metallographic examination is a traditional technology. In this paper, etching method of macro inspection of metallographic was set as an example. Through analyzing the example by Su-Field Model and Physical Contradictions, we convert a practical problem into a TRIZ problem, and find out the final solution from many TRIZ solutions. By the usage, advantage of TRIZ is illuminated.
\end{abstract}

\section{Introduction}

Metallographic examination is one of the very important methods for the industry of metallic material, and it is the important part of examination of the raw metallic material or the metallic product ${ }^{[1]}{\text {. } \text { iu }^{[2]}}^{[3}$ did some researches on the polishing procedure of metallography, invented the electrolysis mix machinery polish craft. Wei ${ }^{[3]}$ did some researches on the sample making of metallography, concluded some special methods for unusual metallic material sample making. Researches in the field of metallographic examination procedure were abundant inside or outside our countries. TRIZ is Theory of Inventive Problem Solving, which can shorten the period of invention, and also make the invention more foreseeable $\mathrm{e}^{[4,5]}$.some researches about efficiency increasing without cost increasing were done by many people. In these fields that solving the problem with obvious contradiction, TRIZ afford the theory support to us and help people exploit their thinking so that people can find out solutions from the problem which seem to be impossible. At the mean time,we tries to demonstrate the TRIZ by case study.But the usage of TRIZ in metallographic examination was not very frequently. So it is significant to do some researches with TRIZ in this field.

\section{Case Study}

This article has chosen a small sized enterprise as a case to study the general operation of macroscopic metallographic examination, then illuminates how to use TRIZ to make some improvement in this case. Macroscopic metallographic examination is mainly use for analyzing the quality of the metallic weld joint or inspecting the defects of the raw material. The existing procedure of macroscopic metallographic examination is shown in Fig. 1.

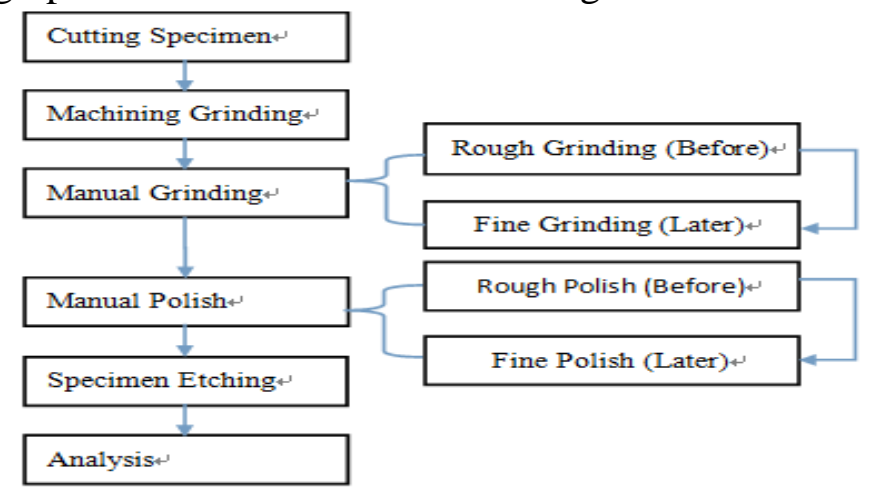

Fig. 1 Procedure of macroscopic metallographic examination 
As shown in Fig.1, macroscopic metallography examination was separated into six steps. However, the most important step is the 6th step (Observing and Analyzing). The 1st step to 5th step was regarded as the pretreatment of the metallographic examination, and the engineer or the technician make decision after finishing the 6th step. In the 6th step, engineer or technician has to use his knowledge to find out the essential problem from the superficial phenomenon of the specimen. To the point of view of a third-party detection institution, the above step is the value-added procedure. For many other manufacture companies, the 6th step is also the most significant one.

Elapsed time of macroscopic metallographic examination is shown in Table 1 (Note: data in Table 1 is the average value which summarized by the writer who worked in this field for a long time). It seems that except for the 6th step, the other steps cost much time of the whole procedure. But we know that the 6th step is the value-added or the significant one. So we try to innovate some new method to decrease the time cost of step 1-step 5.

Table 1 Time elapsed of each procedure

\begin{tabular}{ccccccc}
\hline Procedure & $\begin{array}{c}\text { Cutting } \\
\text { specimen }\end{array}$ & $\begin{array}{c}\text { Mechanical } \\
\text { grinding }\end{array}$ & $\begin{array}{c}\text { Manual } \\
\text { grinding }\end{array}$ & $\begin{array}{c}\text { Manual } \\
\text { polishing }\end{array}$ & $\begin{array}{c}\text { Specimen } \\
\text { etching }\end{array}$ & $\begin{array}{c}\text { Observing \& } \\
\text { Analyzing } \\
\text { (Added-value } \\
\text { process) }\end{array}$ \\
\hline $\begin{array}{c}\text { Time elapsed } \\
\text { (min) }\end{array}$ & $1 \sim 3$ & $1 \sim 3$ & $5 \sim 7$ & $2 \sim 5$ & $\mathbf{1} \sim \mathbf{3}$ & $3 \sim 5$ \\
\hline
\end{tabular}

\section{Solution of TRIZ}

To research and solve the above problem, the Su-Field model and the Physical Contradiction can be used. Step Manual Grind and Step Manual polish are the objects to be improved. The best result is that these two steps can be omitted.

The purpose of Step Manual Grind and Step Manual Polish are wiping off the machining tracks which on the surface of the macroscopic examination specimen. So the specimen can be etched easily next step. In general, low concentration acid is suitable for etching the general metallic material in room temperature. But if we omit the Step Manual Grind and Step Manual Polish and etch the specimen directly, the machining tracks will remain there and affect the judgment of the inspectors.

According to the situation above, relative elements can be found to build the TRIZ Su-Field model. As shown in Fig.2, S1 represents specimen, S2 represents etching agent, F represents chemical field. According to Fig 3-3, we must think that the system belongs to insufficient mode, and 76 Standard Solution can be adopted to solve the problem, and then improvement scheme will be generate.

Solution 1 (Heating method): Add another Field (F2) to intensify the useful effect

According to the hints of 76 Standard Solution, another exterior field (F2) can be added to the model to intensify the useful effect. Combine with the actual situation in this case, the added field can be realized by the way of heating. The specific operations are as follow. Use the heating equipment to heat the etching agent, then wipe the surface of the specimen with the heated etching agent or immerse the specimen into the heated etching agent. Thereafter the surface of the specimen would be etched satisfactorily (The metallic structure or the defects will be exposed clearly). The improved Su-Field model is shown in Fig.3.

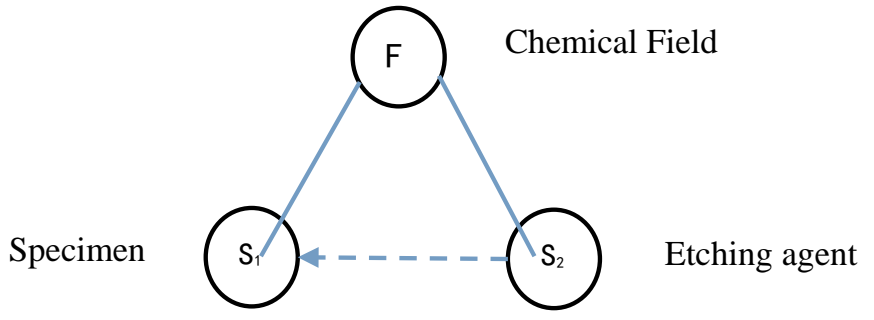

Fig.2 Specimen and Etching agent Su-Field model 


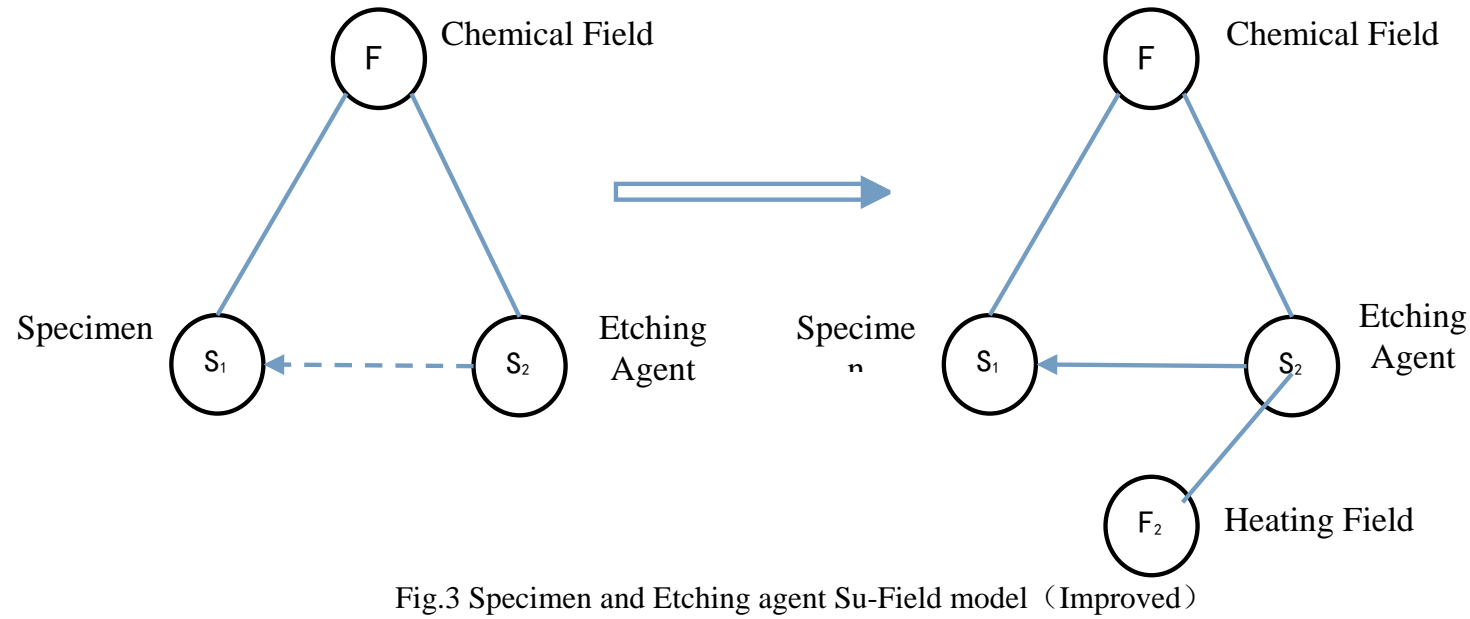

Solution 2 (Electrolyzing method): Plus another substance (S3) and add another Field (F2) to intensify the useful effect

According to the hint of 76 Standard Solution (Add another field to intensify the useful effect), and combine with the actual situation in this case, another field can be realized by the way of electrolyzing. It means we can change the chemical etching into electrochemical etching with electric current. Because of the electric current, the rate of etching will be increased obviously. The specific operations are as follow. Add an auxiliary cathode as S3, such as stainless steel and so on. Then connect the specimen (positive pole)-S1 and the auxiliary cathode-S3, after that apply the current to this loop to make the electric field (F2). Thus the strength of the etching agent will be strengthened and the surface of the specimen will be etched very well. The improved Su-Field model is shown in Fig.4. Solution sketch is shown in Fig.5.

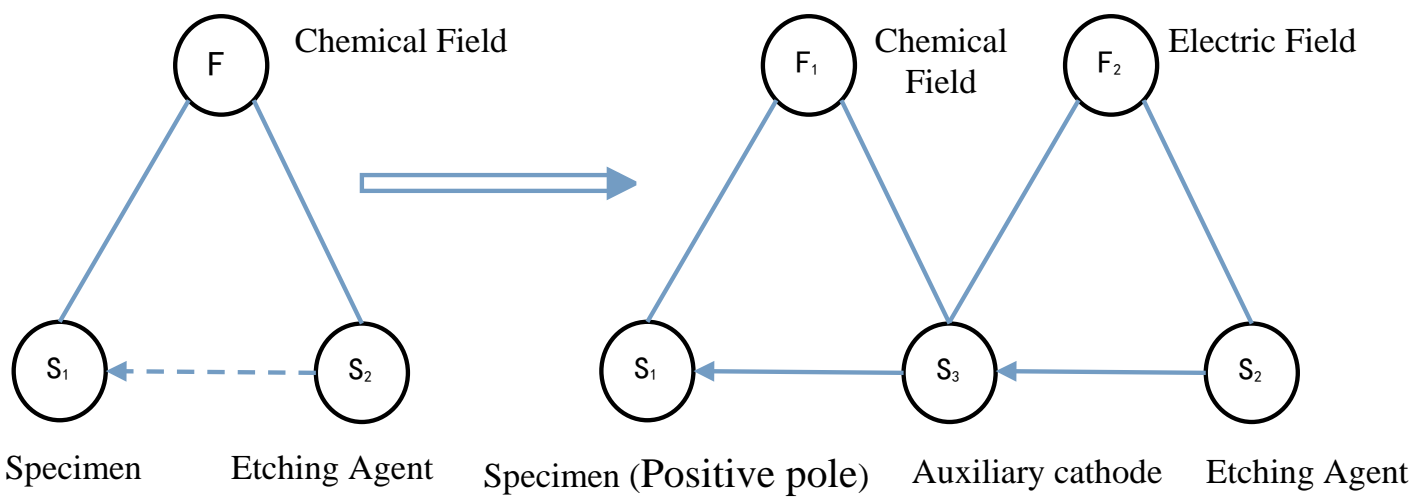

Fig.4 Specimen and Etching agent Su-Field model（Improved）

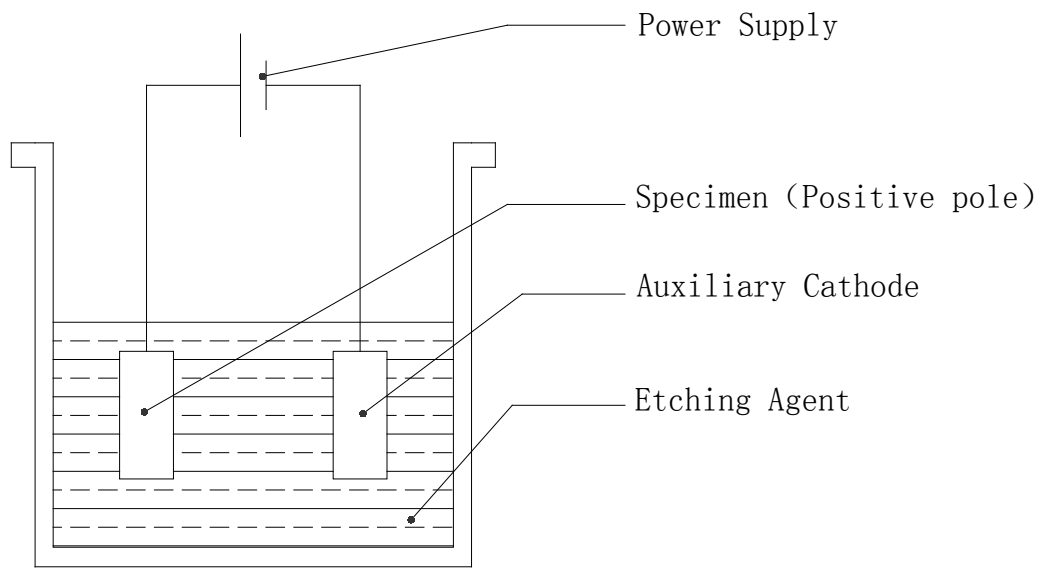

Fig.5 Sketch map of Solution 2

Solution 3 (Applying of Physical Contradiction) 
According to the solution principle of Physical Contradiction, Time separation principle and the corresponding inventive principle-No.16 (Partial of excessive actions) and No.21 (Skipping) can be used. First, from the inspiration of time separation principle, we can separate the etching process into two segments, one is pre-etching and the other is final-etching. In this way, two independent etching procedures are separated by time sequence. Second, from the hint of inventive principle No.16 (Partial of excessive actions), high concentration etching agent should be used to etch the surface of the specimen. It can eliminate all the machining tracks though some over-corrosion may be made. Third, from the inventive principle No.21 (Skipping), we should shorten the contact duration of the specimen and the high concentration etching agent because the high concentration may do harm to the surface of the specimen.

Synthesizes time separation principle and inventive principle No.16 \& 21, final scheme can be made. That is one kind of etching agent, two kinds of concentration. Use the high concentration etching agent first to process the etching first time, but in this procedure, the duration should be as short as possible, once the machining tracks are eliminated, the process should be stopped. Then use the low concentration one to wipe the surface of the specimen again. Since the concentration is low, the corrosivity of the etching agent is not very obvious and it may not etch the specimen seriously. However the low concentration etching agent can remove the corrosion products which generated just before. After this procedure, the corrosion products will be eliminated thoroughly and the macroscopic structure and the metallic defects will be appeared clearly. By this way the procedures of TRIZ innovative solution are finished.

\section{Summary}

Three solutions above are all available, but there are differences among them. The mainly idea of this article is find out an efficiently and effectively method to improve the production efficiency for the small and medium-sized enterprises. Thus one optimum solution of three should be chosen.

Solution 1 is easy to carry out. Because base on the original equipments, it only needs to add a heating equipment. But consider that the etching agents are acidic or alkaline corrosive liquid, and these liquid will become more dangerous after being heated. Thus it will be stricter with the operators, and then the cost of enterprise has to increase without question. Such of staff training, safety and so on.

Solution 2 need not heat etching agent, but it is more complicated than Solution 1. Because Solution 2 need wire to connect the specimen and the auxiliary cathode, besides it need a constant current and voltage power supply equipment. Furthermore, the operators should master the electronic knowledge and operating. From the point of enterprise, cost of human resource and equipment cost must be increased.

Solution 3 is the simplest. As described above, no equipments or other kinds of etching agent was added. What we need is that make up one etching agent into two concentrations, one is low and the other is high. From the point enterprise, it is no need to make any adjustment. The original personnel and equipments are qualified to do the job. Thus the solution 3 should be considered to be the best.

\section{Acknowledgment}

This project is supported by open project of laboratory of innovation method and decision management system of Guangdong province (2011A060901001-03B), national natural science foundation of China (71071059, 71301054), training plan of Guangdong province outstanding young teachers in higher education institutions (Yq2013009).

\section{References}

[1] Y. X. Hu: Practical Technology of Metallographic Examination. China Machine Press, 2010

[2] X. J. Liu, J. D. Xiao, H. Q. Xu: New Technology \& New Process, No.4(1997), P43-44

[3] J. Z. Wei, G. H. Wang, B. Zong: PTCA(PART:A PHYS.TEST.),Vol.42,No.3(2006),P154-156

[4] W. Y. Zhao. H. G. Zhang, R. H. Tan: Journal of Engineering Design, Vol .15,No.2(2008)

[5] L. S. Wang. TRIZ Innovation Theory and Application Principle. Science Press, Beijing, 2010 